\title{
DUALITY METHODS AND PERTURBATION OF SEMIGROUPS
}

\author{
BY R. T. MOORE ${ }^{1}$ \\ Communicated by M. H. Protter, February 15, 1967
}

1. Introduction. In [5], the author announced several theorems applying the semi-inner product methods of Lumer and Phillips [4] to the perturbation theory of one-parameter holomorphic contraction semigroups on Banach spaces. This note extends the methods to a perturbation theorem of Trotter [9], with proofs, and announces generalizations to locally convex spaces. (See also Kato [3].)

2. Generation theorem, $\phi$-sectorial operators. Let $\mathfrak{X}$ be a complex Banach space, and let $[]:, \mathfrak{X} \times \mathfrak{X} \rightarrow \boldsymbol{C}$ be a semi-inner product for $\mathfrak{X}$, in the sense of [4]: (i) for all $v \in \mathfrak{X}, u \rightarrow[u, v]$ is a linear functional on $\mathfrak{X}$, (ii) $[u, u] \geqq 0$ for all $u \in \mathfrak{X}$, with $\|u\|=[u, u]^{1 / 2}$, and (iii) $|[u, v]|$ $<\|u\|\|v\|$.

Definition 1. A linear operator $A$ with domain $D(A) \subset \mathfrak{X}$ is $\phi$ sectorial for $0 \leqq \phi \leqq \pi / 2$ iff for every $u \in \mathscr{D}(A)$,

$$
\tan \phi|\operatorname{Im}[A u, u]| \leqq-\operatorname{Re}[A u, u] \geqq 0 .
$$

Every $\phi_{1}$-sectorial operator is $\phi_{2}$-sectorial for all $\phi_{2} \leqq \phi_{1}$, and every 0 -sectorial operator is dissipative $(\operatorname{Re}[A u, u] \leqq 0$ as in [4]). If $\phi=\pi / 2$, replace the first inequality by $\operatorname{Im}[A u, u]=0$. If $\Delta_{\phi}=\{z \mid \pi$ $\geqq|\arg z| \geqq \pi / 2+\phi\}$, and $W(A)=\{[A u, u] \mid u \in \mathscr{D}(A),\|u\|=1\}$ is the numerical range of $A$ then $A$ is $\phi$-sectorial iff $\Delta_{\phi} \supset\{W(A)\}$ - (obvious when sketched).

Definition 2. A one-parameter semigroup $T$ is in the family $\mathrm{CH}(\phi)$ of holomorphic contraction semigroups on the sector $S_{\phi}$ $=\{z|| \arg z \mid \leqq \phi\}$ iff

(a) $T$ is a homomorphism of the additive semigroup of $S_{\phi}$ into the multiplicative semigroup $\mathfrak{C}(\mathfrak{X})$ of all contraction operators on $\mathfrak{X}$ $(\|T(z)\| \leqq 1)$,

(b) $z \rightarrow T(z)$ is a holomorphic function from int $\left(S_{\phi}\right)$ toe $(\mathfrak{X}) \subset \mathfrak{L}(\mathfrak{X})$, the Banach algebra of bounded operators on $\mathfrak{X}$ (see [2, Chapter 5]), and

(c) (slightly redundant) for all $u \in \mathfrak{X}$, the map $z \rightarrow T(z) u$ is continuous from $S_{\phi}$ into $\mathfrak{X}$.

\footnotetext{
${ }^{1}$ Research supported in part by NSF Contract GP 5585.
} 
(d) Then the (infinitesimal) generator $A$ of $T$ is defined, for all $u \in \mathfrak{X}$ where the limit through real $h$ exists, by

$$
A u=\lim _{h \backslash 0} h^{-1}\{T(h) u-u\} .
$$

THEOREM 1. An operator $A$ is the infinitesimal generator of a $C H(\phi)$ semigroup $T$ iff

(a) $A$ is closed, densely defined, and $\phi$-sectorial, and

(b) $\rho(A) \cap\left(C \sim \Delta_{\phi}\right) \neq \varnothing$, where $\rho(A)$ is the resolvent set.

LemmA A. $A$ is $\phi$-sectorial iff $e^{i \theta} A$ is dissipative for all $0 \leqq|\theta| \leqq \phi$ $\leqq \pi / 2$.

ProOF.

$$
\begin{aligned}
\operatorname{Re}\left[e^{i \theta} A u, u\right] & =\operatorname{Re}\left(e^{i \theta}[A u, u]\right) \\
& =\cos \theta \operatorname{Re}[A u, u]-\sin \theta \operatorname{Im}[A u, u]
\end{aligned}
$$

Since all such $\cos \theta$ are positive, $e^{i \theta} A$ is dissipative and (3) is negative for all $u \in D(A)$ iff, dividing by $-\cos \theta$,

$$
0 \leqq-\operatorname{Re}[A u, u] \geqq \operatorname{Im}[A u, u] \tan \theta .
$$

Since $\tan \theta$ is monotone increasing, this holds for all $\theta$ in the specified range iff it holds for $\theta= \pm \phi$, depending upon the sign of $\operatorname{Im}[A u, u]$. This last is equivalent to (1).

Lemma B. Suppose $A$ is $\phi$-sectorial, $u \in \mathfrak{D}(A)$, and $z \notin \Delta_{\phi}$. Then

$$
\|(z-A) u\| \geqq d\left(z, \Delta_{\phi}\right) u,
$$

where $d\left(z, \Delta_{\phi}\right)$ is the distance from $z$ to $\Delta_{\phi}$.

Proof. Clearly if $|\arg z| \leqq \phi, d\left(z, \Delta_{\phi}\right)=|z|$. Then

$$
\begin{aligned}
\|(z-A) u\| & =\|\exp (i \arg z)(|z|-\exp (-i \arg z) A) u\| \\
& =\|(|z|-\exp (-i \arg z) A) u\| .
\end{aligned}
$$

But here $\exp (-i \arg z) A$ is dissipative by Lemma 2, and a calculation from [4] yields for any dissipative $B$ and $z_{0} \in C$ :

$$
\begin{aligned}
\operatorname{Re}\left(z_{0}\right)\|u\|^{2} & =\operatorname{Re}\left[z_{0} u, u\right] \leqq \operatorname{Re}\left(\left[z_{0} u, u\right]-[B u, u]\right) \\
& \leqq\left|\left[\left(z_{0}-B\right) u, u\right]\right| \leqq\left\|\left(z_{0}-B\right) u\right\|\|u\| .
\end{aligned}
$$

Cancelling $\|u\|$ and applying this with $z_{0}=|z|, B=\exp (-i \arg z) A$,

$$
\|(z-A) u\| \geqq \operatorname{Re}\left(z_{0}\right)\|u\|=|z|\|u\|=d\left(z, \Delta_{\phi}\right)\|u\|
$$

by (6). 
But if $\phi \leqq|\arg z| \leqq \pi-\phi$, trigonometry shows that

$$
d\left(z, \Delta_{\phi}\right)=\cos (\arg z-\phi)|z| .
$$

Then $e^{-i \phi} A$ is dissipative by Lemma 2 and by the same procedure with $z_{0}=\exp (i(\arg z-\phi))|z|, B=e^{-i \phi} A$,

$$
\begin{aligned}
\|(z-A) u\| & =\left\|e^{i \phi}\left\{\exp (i(\arg z-\phi))|z|-e^{-i \phi} A\right\} u\right\| \\
& =\left\|\left(\exp (i(\arg z-\phi))|z|-e^{-i \phi} A\right) u\right\| \\
& \geqq \operatorname{Re}(\exp (i(\arg z-\phi))|z|)\|u\| \\
& =\cos (\arg z-\phi)|z|\|u\|=d\left(z, \Delta_{\phi}\right)\|u\| .
\end{aligned}
$$

Lemma C. If (5) holds and $A$ is closed $\rho(A)$ either is disjoint from $\mathrm{C} \sim \Delta_{\phi}$ or contains $\mathrm{C} \sim \Delta_{\phi}$ (the complement of $\Delta_{\phi}$ in $\mathrm{C}$ ).

Proof. If $z_{0} \in \rho(A) \cap\left(C \sim \Delta_{\phi}\right)$ then (5) yields

$$
\left\|\left(z_{0}-A\right)^{-1}\right\| \leqq d\left(z_{0}, \Delta_{\phi}\right)^{-1} \text {. }
$$

Then the argument of [10, Theorem VIII.2.1], with this estimate, shows that for $z$ in the open disc about $z_{0}$ tangent to $\Delta_{\phi}\left(\left|z_{0}-z\right|\right.$ $\left.<d\left(z_{0}, \Delta_{\phi}\right)\right), z \in \rho(A)$, with the Neumann expansion

$$
(z-A)^{-1}=\sum_{k=0}^{\infty}\left(z_{0}-z\right)^{k}\left(z_{0}-A\right)^{-(k+1)} .
$$

Any nonempty subset of $C \sim \Delta_{\phi}$ containing a $\Delta_{\phi}$-tangent disc about each of its members exhausts $C \sim \Delta_{\phi}$ (induction).

Lemma D (Hille). Let $T$ be any strongly continuous semigroup on $S_{\phi}$ whose restriction to int $\left(S_{\phi}\right)$ is in $H(\phi, \phi)$ ([2, Definition 10.6.1, p. 325]), and whose generator is $A$. Then for $|\theta| \leqq \phi, t \rightarrow T_{\theta}(t)=T\left(e^{i \theta} t\right)$ is a semigroup of class $C_{0}$ with generator $A_{\theta}=e^{i \theta} A$. If $T \in C H(\phi)$, then $T_{\theta} \in C H(0)$ and $e^{i \theta} A$ is dissipative for $|\theta| \leqq \phi$.

Proof. The argument of Lemma 10.6.2 and the first part of the proof of Theorem 12.8.1 in [2] yields finite constants $M_{\phi}$ and $\omega_{\phi}$ with

$$
\left\|T\left(e^{i \theta} t\right)\right\| \leqq M_{\phi} e^{\omega t} \text {. }
$$

Then the deformation-of-contours argument on page 384 of [2] leads to the following rewording of 12.8 .4 :

$$
(\lambda-A)^{-1} u=e^{i \theta} \int_{0}^{\infty} e^{-\lambda e^{i \theta}} T\left(e^{i \theta} t\right) u d t=e^{i \theta}\left(e^{i \theta} \lambda-A_{\theta}\right)^{-1} u \equiv v
$$

For such a $v$ (these exhaust $\mathfrak{D}(A)=\mathfrak{D}\left(A_{\theta}\right)$ ) 


$$
\begin{aligned}
e^{i \theta} A v=-e^{i \theta}(\lambda-A) v+e^{i \theta} \lambda v & =-e^{i \theta} u+e^{i \theta} \lambda v \\
& =-e^{i \theta} u+\left(e^{i \theta} \lambda-A_{\theta}\right) v+A_{\theta} v \\
& =-e^{i \theta} u+e^{i \theta} u+A_{\theta} v=A_{\theta} v,
\end{aligned}
$$

substituting twice and cancelling.

If $T$ consists entirely of contractions, so does $T_{\theta}$, hence Theorem 3.2 of [4] applies to prove that $e^{i \theta} A=A_{\theta}$ is dissipative.

Proof of Theorem 1. If $T \in C H(\phi)$, every $e^{i \theta} A$ is dissipative by Lemma $\mathrm{D}$, for $|\theta| \leqq \phi$, so $A$ is $\phi$-sectorial by Lemma $\mathrm{A}$. By Theorem 3.2 of [4] again, since $T_{0}$ is a contraction semigroup, $A_{0}=A$ is closed, densely defined, and has $1 \in \rho(A) \cap C \sim \Delta_{\phi}$. Hence (a) and (b) hold.

Suppose (a) (especially $A \phi$-sectorial) and (b), so that by Lemmas $\mathrm{B}$ and $\mathrm{C}$ and equation (5),

$$
\left\|(z-A)^{-1}\right\| \leqq d\left(z, \Delta_{\phi}\right)^{-1} .
$$

Then Hille's Theorem 12.8 .1 of [2] shows that $A$ generates a $H(-\phi, \phi)$ semigroup $T^{h}$ on int $\left(S_{\phi}\right)$. Applying Lemma $\mathrm{D}$ to closed subsectors, and Lemma 1 to see that $e^{i \theta} A$ is dissipative, it follows that all $T^{h}\left(e^{i \theta} t\right)$ are contractions by Theorem 3.2 of [4]. It remains to show that the contraction semigroups generated by $e^{ \pm i \phi} A$ extend $T^{h}$ to all of $S_{\phi}$, forming a $C H(\phi)$ semigroup $T$. All $T_{\theta}$ generated by $e^{i \theta} A$ for $|\theta| \leqq \phi$ leave the common $D(A)=D\left(e^{i \theta} A\right)$ invariant, are differentiable on it and commute with $A$ (Theorem 10.3 .3 of [2]). If $u \in \mathscr{D}(A)$,

$$
\begin{aligned}
T_{ \pm \phi}(t) u-T_{\theta}(t) u & =\int_{0}^{t} \frac{d}{d s}\left(T_{ \pm \phi}(s) T_{\theta}(t-s) u\right) d s \\
& =\int_{0}^{t} T_{ \pm \phi}(s)\left(e^{ \pm \phi} A-e^{i \theta} A\right) T_{\theta}(t-s) u d s \\
& =\left\{e^{ \pm i \phi}-e^{i \theta}\right\} \int_{0}^{t} T_{ \pm \phi}(s) T_{\theta}(t-s) A u d s .
\end{aligned}
$$

Since the $T$ 's are contractions, the last integral is smaller than $t\|A u\|$ and, as $\theta \rightarrow \pm \phi, T_{\theta}(t) u \rightarrow T_{ \pm \phi}(t) u$ uniformly on $t$-compacta, allowing a continuous extension of $z \rightarrow T^{h}(z) u$ to $S_{\phi}$. By $3-\epsilon$, this extends to all $u \in X$, and the semigroup property extends by limits as well, to create a $T \in C H(\phi)$.

\section{The perturbation theorems.}

Theorem 2. (a) If $A$ and $B$ are $\phi$-sectorial, and $\alpha$ and $\beta$ nonnegative, then $D=\alpha A+\beta B$ is $\phi$-sectorial (see [8]). 
(b) If $\left\{A_{\alpha} \mid \alpha \in I\right\}$ is a net of $\phi$-sectorial operators, and $D$ is defined, for all $u$ where the limit exists in $\cap\left\{D\left(A_{\alpha}\right) \mid \alpha \in I\right\}$, by $D u=\lim A_{\alpha} u$, then $D$ is $\phi$-sectorial.

Proof. (a) If $u \in D(A) \cap D(B), \quad[(\alpha A+\beta B) u, u]=\alpha[A u, u]$ $+\beta[B u, u]$. Then $W(\alpha A+\beta B) \subset \alpha W(A)+\beta W(B) \subset \Delta_{\phi}$ since $\Delta_{\phi}$ is a cone; the same applies for closures since $\Delta_{\phi}$ is closed (Def. 1 et seq.).

(b) $[D u, u]=\left[\left(D-A_{\alpha}\right) u, u\right]+\left[A_{\alpha} u, u\right]$ and $\left|\left[\left(D-A_{\alpha}\right) u, u\right]\right|$ $\leqq\left\|\left(D-A_{\alpha}\right) u\right\|\|u\| \rightarrow 0$; so $[D u, u]=\lim \left[A_{\alpha} u, u\right]$, and the same applies to real and imaginary parts, so (1) for $D$ follows from (1) for the $A_{\alpha}$.

Theorem 3. Suppose $D$ in Theorem 2 (a) or (b) is densely defined, and for some $z_{0} \notin \Delta_{\phi}$, range $(z-D)$ is dense. Then $\bar{D}$ exists and generates a $\mathrm{CH}(\phi)$ semigroup.

Proof. All $e^{i \theta} D$ for $|\theta| \leqq \phi$ are dissipative by Lemma A. Theorem 3.3 of [4] insures that $\bar{D}$ exists, and an easy modification of the proof of their Lemma 3.4 shows that a new semi-inner product can be chosen making all $e^{i \theta} \bar{D}$ dissipative at once, so $\bar{D}$ becomes $\phi$-sectorial. Then as in Theorem VIII.1.1 of [10, p. 209], $z_{0} \in \rho(\bar{D})$ and Theorem 1 applies.

The following can supply the range condition:

(DA) $D$ has a densely defined dissipative adjoint $D^{*}$; e.g. in (b) the net $\left\{A_{\alpha}^{*} \mid \alpha \in I\right\}$ consists of dissipative operators converging on a dense subset of $\mathfrak{X}^{*}$ (see Corollary 3.2 in [4]).

(G) In (a), $D(A) \subset D(B)$ and for some $a<1$ and $b \geqq 0,\|B u\|$ $\leqq a\|A u\|+b\|u\|$ for all $u \in \mathscr{D}(A)$ (see [1]).

THEOREM 4. If $\left\{A_{\alpha} \mid \alpha \in I\right\}$ is a net of generators of $C H(\phi)$ semigroups $T_{\alpha}$ with a limit $D$ satisfying Theorem 3 (or (DA)) then $\bar{D}$ generates a $C H(\phi)$ semigroup $T$ which is the uniform strong limit on compacta in $S_{\phi}$ of the $T_{\alpha}$.

Proof. We already know that $T$ exists and that $C \sim \Delta_{\phi} \subset \rho(\bar{D})$. The usual argument for the Trotter-Kato theorem (see [10, p. 270$271])$ can then be shortened considerably because the limit semigroup $T$ and limit resolvents $(z-\bar{D})^{-1}$ are already known to exist, but essentially the same reasoning is used to prove uniform convergence on compacta. (The novelty lies in the treatment of the cases $\phi \neq 0$ and the avoidance of ergodic theorems for pseudoresolvents. For another proof, see [7].)

4. Generalizations. If a family $\Gamma$ of seminorms $p$ calibrates (gives a locally convex topology to) a complex vector space $\mathfrak{X}$, there is a 
Lumer structure $\Lambda=\left\{[,]_{p} \mid p \in \Gamma\right\}$ for $\mathfrak{X}$ consisting of indefinite semiinner products with $[u, u]_{p}^{1 / 2}=p(u) . A$ is $\phi$-sectorial for $\Lambda$ if it is $\phi$ sectorial for every $[,]_{p}$, and $T(z)$ is a contraction iff for all $u \in \mathfrak{X}$ and $p \in \Gamma, p(T(z) u) \leqq p(u)$. If "holomorphic" is taken to mean " $z \rightarrow\left\langle u^{*}, T(z) u\right\rangle$ is holomorphic for all $u^{*} \in \mathfrak{X}^{*}$," the entire theory presented above can be generalized. Furthermore, every equicontinuous semigroup creates a $\Gamma$ for which it is a contraction semigroup (see [6]), and it turns out that the results given in Chapter IX of [10], along with several new theorems, can be obtained in this way. Details will appear in [7].

\section{BiBLIOGRAPHY}

1. K. Gustafson, A perturbation lemma, Bull. Amer. Math. Soc. 72 (1966), 334338.

2. E. Hille, and R. S. Phillips, Functional analysis and semigroups, Amer. Math. Soc. Colloq. Pub., vol. 31 Amer. Math. Soc., Providence, R.I., 1957.

3. T. Kato, Perturbation theory for linear operators, Springer-Verlag, New York, 1966, (see pp. 495 and 502).

4. G. Lumer and R. S. Phillips, Dissipative operators in a Banach space, Pacific J. Math. 11 (1961), 679-698.

5. R. T. Moore, Duality methods in the perturbation of holomorphic semigroups, Notices Amer. Math. Soc. 13 (1966), 554 (Abstract 636-98).

6. - Contractions, equicontinuous semigroups, and Banach algebras of operators on locally convex spaces, (in preparation).

7. - Contractions, perturbations, and Lumer structures on locally convex spaces, (in preparation).

8. E. Nelson, Feyman integrals and the Schrödinger equation, Appendix B, J. Math. Phys. 5 (1964), 332-343.

9. H. F. Trotter, Approximation of semigroups of operators, Pacific J. Math. 8 (1958), 887-919.

10. K. Yosida, Functional analysis, Academic Press, New York, 1965.

University of California, Berkeley 